\title{
Hukuhara's problem for equations of evolution
}

By

\section{Setuzô YosidA*}

\section{$\S$ Introduction.}

In the former work [12] we have investigated boundary value problem formulated with Hukuhara's data for parabolic system. Now in this paper, we shall treat initial value problem formulated with Hukuhara's data, or shortly, Hukuhara's problem for equations of evolution.

In $\S 1$ we shall consider a system of equations of evolution

$$
\partial_{t} \boldsymbol{u}(t, x)=\sum_{|\boldsymbol{k}| \leqq p} A_{\boldsymbol{k}}(t) \boldsymbol{D}^{\boldsymbol{k}} \boldsymbol{u}(t, \boldsymbol{x})+\boldsymbol{b}(t, \boldsymbol{x}),
$$

and find a solution $\boldsymbol{u}(t, \boldsymbol{x})=\left\{u_{1}(t, \boldsymbol{x})\right)$ of it defined for $0 \leqq t \leqq T_{0}$

$$
\left(\begin{array}{c}
u^{2}(t, \boldsymbol{x}) \\
\vdots \\
u_{N}(t, \boldsymbol{x})
\end{array}\right)
$$

and satisfying

$$
u_{j}\left(t_{j}, \boldsymbol{x}\right)=\varphi_{j}(\boldsymbol{x}), 0 \leqq t_{j} \leqq B_{0}\left(\leqq T_{0}\right), j=1,2 \cdots, N,
$$

for a properly chosen constant $B_{0}$.

We shall apply Fourier transformation $\mathfrak{F}_{x}$ with respect to the variables $\boldsymbol{x}=\left(x_{1}, x_{2}, \cdots, x_{m}\right)$ to the system (0.1) and transform the problem into a Hukuhara's prcblem for ordinary differential equations, which can be solved under a suitable assumption.

In $\S 2$ we shall investigate a system of special type, which can be treated under a weaker assumption than the former one.

In $\S 3$ we shall treat Hukuhara's problem for convolution equations, which can be solved by the same method as in the preceding sections.

* Univ. of Tokyo and Research Inst. Math. Sci., Kyoto Univ., Received July. 29, 1966. 
In the last section we shall consider Hukuhara's problem for a semi-separate symmetric hyperbolic system. This is a system of differential equations of which the principal part consists of separate symmetric hyperbolic systems. Partial differential equations of "semiseparate type" have been investigated by severdl authors. See for example T. Kusano [4], [5] and references in them. K. Akô [1] called them "semi-decomposable type".

We shall prove existence, uniqueness and stability of solution of such a system.

The author wishes to express his hearty thanks to Prof. M. Hukuhara and Prof. K. Yosida for their helpful advice and incessant encouragement.

\section{§1. Hukuhara's problem for general equations of evolution.}

1.1. We use following notations; bold letters stand for vectors;

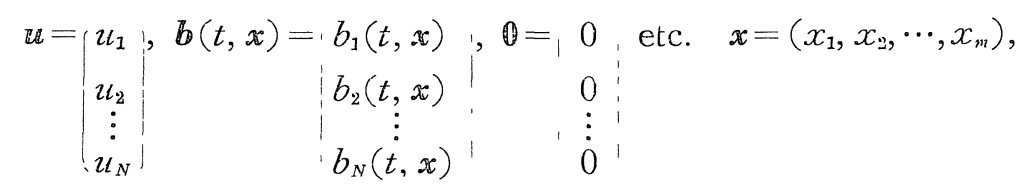

$\boldsymbol{\sigma}=\left(\sigma_{1}, \sigma_{2}, \cdots, \sigma_{m}\right), \sigma x=\sigma_{1} x_{1}+\sigma_{2} \varkappa_{2}+\cdots+\sigma_{m} x_{m},|\boldsymbol{\sigma}|=\left|\sigma_{1}\right|+\left|\sigma_{2}\right|+\cdots+\left|\sigma_{m}\right|$, $\mathbb{J}_{\varepsilon}=\left(k_{1}, k_{2}, \cdots k_{m}\right), \quad k_{J}^{\prime} \mathrm{S}$ are nonnegative integers. $\left|k_{\varepsilon}\right|=k_{1}+k_{2}+\cdots+k_{m}$, $\partial_{t}=\partial / \partial t, \quad \partial_{j}=\partial / \partial x_{j}, \quad D_{j}=-i \partial_{j}, \quad \mathbb{D}^{k}=D_{1}^{k_{1}} D_{2}^{k_{2}} \cdots D_{m}^{k_{m}} . \quad t, \quad x_{j}, \sigma_{j}$ are real variables. $u_{j}(t, \boldsymbol{x}), b_{j}(t, \boldsymbol{x}), \varphi_{j}(\boldsymbol{x})$ etc. are in general complex valued functions.

Consider the following equations;

$$
\partial_{t} \mathbb{u}=\sum_{|k| \leqq p} A_{k}(t) \mathbb{D}^{k} \mathbb{u}+\boldsymbol{b}(\dot{t}, \mathfrak{x}),
$$

in which $A_{k}(t)$ 's are $N \times N$ matrices. $\quad(t, x)$ is an ' $N$-dimensional vector. We shall solve Hukuhara's problem for this system and find as solution $\boldsymbol{u}(t, \boldsymbol{x})$, defined for $0 \leqq t \leqq T_{0}$ and satisfying the initial condition with Hukuhara's data

$$
u_{j}\left(t_{j}, \boldsymbol{x}\right)=\varphi_{j}(\boldsymbol{x}), 0 \leqq t_{j} \leqq B_{0}\left(\leqq T_{0}\right), j=1,2, \cdots, N
$$


or in vector form

$$
\left.\boldsymbol{u}(t, \boldsymbol{x})\right|_{H}=\varphi(\boldsymbol{x}) .
$$

We apply to $\boldsymbol{u}(t, \boldsymbol{x})$ Fourier transformation $\mathfrak{F}_{\boldsymbol{x}}$ with respect to the variable $x$ formally and obtain

$$
\begin{aligned}
& \boldsymbol{v}(t, \boldsymbol{\sigma})=\mathfrak{F}_{\boldsymbol{x}} \boldsymbol{u}(t, \boldsymbol{x}) \equiv \int \exp (-i \boldsymbol{\sigma} \boldsymbol{x}) \boldsymbol{u}(t, \boldsymbol{x}) d \boldsymbol{x}, \\
& \partial_{t} \boldsymbol{v}(t, \boldsymbol{\sigma})=\sum_{|\boldsymbol{k}| \leq p} A_{\boldsymbol{k}}(t) \boldsymbol{\sigma}^{\boldsymbol{k}} \boldsymbol{v}(t, \boldsymbol{\sigma})+\boldsymbol{b}(t, \boldsymbol{\sigma}), \\
& v_{j}\left(t_{j}, \boldsymbol{\sigma}\right)=\hat{\varphi}_{j}(\boldsymbol{\sigma}), j=1,2, \cdots, N,
\end{aligned}
$$

or

$$
\left.\left(1.5^{\prime}\right) \quad \boldsymbol{v}(t, \boldsymbol{\sigma})\right|_{H}=\hat{\varphi}(\boldsymbol{\sigma}),
$$

in which we used the notations $\hat{b}(t, \sigma)=\mathfrak{F}_{x} \mathfrak{b}(t, x), \hat{\varphi}(\boldsymbol{\sigma})=\mathfrak{F}_{x} \boldsymbol{\varphi}(\boldsymbol{x})$.

We assume;

A) i) The elements $a_{k j l}(t)$ 's of the matrices $A_{k}(t)$ 's are defined and continuous for $0 \leqq t \leqq T_{0}$, satisfy $\left|a_{k j l}(t)\right| \leqq \alpha_{0}$ with some constant $\alpha_{0}$. ii) The components $b_{j}(t, x)$ 's of the vector $b(t, x)$ are defined and integrable in $x$ for fixed $t$, which we denote by the notation $b_{j}(t, \boldsymbol{x}) \in L_{\boldsymbol{x}}^{1}$. The Fourier transforms $\hat{b}_{j}(t, \sigma)$ are continuous with respect to $t$ and satisfy the inequality $\left|\hat{b}_{j}(t, \sigma)\right|=\beta_{0}(\sigma)$ with a function $\beta_{0}(\sigma) \in L_{\sigma}^{1}$ with compact support; $\beta_{0}(\sigma) \equiv 0$ for $|\sigma|>X_{0}$ with a constant $X_{0}$. iii) The components $\varphi_{,}(x)$ 's of the vector $\varphi(x)$ belong to $L_{x}^{1} . \hat{\varphi}_{j}(\sigma)$ 's have compact supports; $\hat{\varphi}_{j}(\mathbb{*}) \equiv 0$ for $|\sigma|>X_{0}$.

1. 2. Since (1.4) is a system of ordinary differential equations including the parameters $\sigma$, we consider the reduced equation of it;

$$
\partial_{t} \boldsymbol{v}(t, \boldsymbol{\sigma})=\sum_{|\boldsymbol{k}| \underline{\underline{1}} \boldsymbol{p}} A_{\boldsymbol{k}}(t) \boldsymbol{\sigma}^{\boldsymbol{k}} \boldsymbol{v}(t, \boldsymbol{\sigma}) .
$$

Let $\boldsymbol{v}^{(j)}(t, \boldsymbol{\sigma}) j=1,2, \cdots, N$ be its fundamental system of solutions satisfying

$$
\boldsymbol{v}^{(j)}(0, \boldsymbol{\sigma})=\boldsymbol{d}^{(j)} \equiv\left(\begin{array}{c}
0 \\
\vdots \\
0 \\
1 \\
0 \\
\vdots \\
0
\end{array}\right)
$$


or in matrix form

$$
\begin{aligned}
& V(0, \boldsymbol{\sigma})=E, \quad \text { where } \\
& V(t, \boldsymbol{\sigma})=\left(\boldsymbol{v}^{(1)}(t, \boldsymbol{\sigma}), \boldsymbol{v}^{(2)}(t, \boldsymbol{\sigma}), \cdots, \boldsymbol{v}^{(N)}(t, \boldsymbol{\sigma})\right) .
\end{aligned}
$$

One particular solution $\boldsymbol{v}^{(0)}(t, \boldsymbol{\sigma})$ of the original system (1.4) is given by the formula

$$
\boldsymbol{v}^{(0)}(t, \boldsymbol{\sigma})=V(t, \boldsymbol{\sigma}) \int_{0}^{t}(V(s, \boldsymbol{\sigma}))^{-1} \hat{\boldsymbol{b}}(s, \boldsymbol{\sigma}) d s .
$$

We notice that from the assumption A) ii) we have $\boldsymbol{v}^{(0)}(t, \sigma) \equiv 0$ for $|\sigma|>X_{0}$.

We try to find a solution $\boldsymbol{v}(\boldsymbol{t}, \boldsymbol{\sigma})$ of $(1.4)$ (1.5) in the form

$$
\left\{\begin{array}{l}
\boldsymbol{v}(t, \boldsymbol{\sigma})=\boldsymbol{v}^{(0)}(t, \boldsymbol{\sigma})+\sum_{i=1}^{N} \boldsymbol{v}^{(j)}(t, \boldsymbol{\sigma}) c_{j}(\boldsymbol{\sigma}) \equiv \boldsymbol{v}^{(0)}(t, \boldsymbol{\sigma})+V(t, \boldsymbol{\sigma}) \boldsymbol{c}(\boldsymbol{\sigma}) \\
\boldsymbol{v}(t, \boldsymbol{\sigma}) \equiv 0 \text { for }|\boldsymbol{\sigma}| \leqq X_{0}, \\
\text { for } \mid>X_{0} .
\end{array}\right.
$$

The condition (1.5) for this $\boldsymbol{v}(t, \sigma)$ becomes

$$
\left(\left.V(t, \sigma)\right|_{H}\right) c(\sigma)=\hat{\varphi}(\sigma)-\left.v^{(0)}(t, \sigma)\right|_{H} \equiv \psi(\sigma),
$$

in which we used the notation

$$
\left.V(t, \boldsymbol{\sigma})\right|_{H} \equiv\left(\begin{array}{cccc}
v_{1}^{(1)}\left(t_{1}, \boldsymbol{\sigma}\right) & v_{1}^{(2)}\left(t_{1}, \boldsymbol{\sigma}\right) & \cdots & v_{1}^{(N)}\left(t_{1}, \boldsymbol{\sigma}\right) \\
v_{2}^{(1)}\left(t_{2}, \boldsymbol{\sigma}\right) & v_{2}^{(2)}\left(t_{2}, \boldsymbol{\sigma}\right) & \cdots & v_{2}^{(N)}\left(t_{2}, \boldsymbol{\sigma}\right) \\
\cdots & \cdots & \cdots & \cdots \\
v_{N}^{(1)}\left(t_{N}, \boldsymbol{\sigma}\right) & v_{N}^{(2)}\left(t_{N}, \boldsymbol{\sigma}\right) & \cdots & v_{N}^{(N)}\left(t_{N}, \boldsymbol{\sigma}\right)
\end{array}\right)
$$

1. 3. We shall give a lower bound of the absolute value of the Hukuhara's determinant $\operatorname{det}\left(\left.V\right|_{H}\right)$.

Consider any matrix $C=\left(c_{j_{k}}\right)_{j, k=1,2, \cdots, N}$ whose elements $c_{j k}$ satisfy $\left|c_{j k}-\delta_{j k}\right| \leqq \varepsilon, j, k=1,2, \cdots, N, \delta_{j k}$ are Kronecker's symbols, $\varepsilon$ is a small positive constant. We can easily give a lower bound of $|\operatorname{det} C|$ as follows;

$$
|\operatorname{det} C| \geqq(1-\varepsilon)^{N}-\varepsilon^{2}(1+\varepsilon)^{N-2} \quad(N !-1) \equiv f(\varepsilon) .
$$

An elementary calculation gives

$$
f(\varepsilon) \geqq f\left(\varepsilon_{0}\right)>\frac{1}{2}-\frac{1}{4}=\frac{1}{4} \text { for } \quad 0 \leqq \varepsilon \leqq \varepsilon_{0},
$$


where $\varepsilon_{0}$ can be taken as

$$
\varepsilon_{0}=\min \left(1-\left(\frac{1}{2}\right)^{1 / N},\left(1 / 2^{N}(N !-1)\right)^{1 / 2}\right) .
$$

To estimate the elements $v_{k}^{(j)}\left(t_{k}, \boldsymbol{\sigma}\right)$ of the matrix $V(t, \boldsymbol{\sigma})$, we define for any fixed $j$,

$$
w_{k}(t, \boldsymbol{\sigma})=v_{k}^{(j)}(t, \boldsymbol{\sigma})-\boldsymbol{\delta}_{j k}, \quad k=1,2, \cdots, N,
$$

or in vector form

$$
\boldsymbol{w}(t, \sigma)=\boldsymbol{v}^{(j)}(t, \sigma)-d^{(j)} .
$$

From (1.6) and (1.7) we have

$$
\begin{aligned}
& \partial_{t} \boldsymbol{w}(t, \sigma)=\sum_{|\boldsymbol{k}| \leqq p} A_{\boldsymbol{k}}(t) \sigma^{\boldsymbol{k}}\left(\boldsymbol{w}(t, \boldsymbol{\sigma})+\boldsymbol{d}^{(j)}\right), \\
& \boldsymbol{w}(0, \boldsymbol{\sigma})=\mathbf{0} .
\end{aligned}
$$

Since $\boldsymbol{w}(t, \boldsymbol{\sigma})$ is an $N$-dimensional complex valued vector, it is equivalent to a $2 N$-dimensional real valued vector, and we can apply the comparison theorem of ordinary differential equations to it. We compare $w_{k}(t, \boldsymbol{\sigma})$ with the solution $z(t)$ of

$$
\begin{aligned}
& \frac{d}{d t} z(t)=2 N \alpha_{0} \sum_{|k| \leqq p} X_{0}^{|k|} z(t)+\alpha_{0}, \\
& z(0)=0
\end{aligned}
$$

and have

$$
\begin{aligned}
\left|v_{k}^{(j)}-\delta_{j k}\right| & =\left|v_{k}\right| \leqq z(t) \\
& =\frac{1}{2 N \sum_{|\boldsymbol{k}| \leqq p} X_{0}^{|k|}}\left\{\begin{array}{c}
\left.\exp \left(2 N \alpha_{0} \sum_{|k| \leqq p} X_{0}^{|k|} t\right)-1\right\} \\
\text { for } 0 \leqq t \leqq T_{0} .
\end{array}\right.
\end{aligned}
$$

Let $B_{0}$ be a constant determined by the equation

$$
\frac{1}{2 N \sum_{|\boldsymbol{k}| \leq p} X_{0}^{|k|}}\left\{\exp \left(2 N \alpha_{0} \sum_{|\boldsymbol{k}| \leq p} X_{0}^{|k|} B_{0}\right)-1\right\}=\varepsilon_{0} .
$$

From (1.13) and (1.20) we have

(1.22) $\left|\operatorname{det}\left(\left.V(t, \boldsymbol{\sigma})\right|_{H}\right)\right| \geqq 1 / 4$ for $0 \leqq t_{j} \leqq B_{0}$, 
which assures us the existence of the inverse matrix $\left(\left.V(t, \sigma)\right|_{H}\right)^{-1}$.

1.4. Now (1.10) can be solved with respect to $\boldsymbol{c}(\boldsymbol{\sigma})$ and we have

$$
\boldsymbol{c}(\boldsymbol{\sigma})=\left\{\begin{array}{lll}
\left.\left.V(t, \boldsymbol{\sigma})\right|_{H}\right)^{-1} \boldsymbol{\psi}(\boldsymbol{\sigma}) & \text { for } & |\boldsymbol{\sigma}| \leqq X_{0} \\
\boldsymbol{0} & \text { for } & |\boldsymbol{\sigma}|>X_{0}
\end{array}\right.
$$

This completes the construction of $\boldsymbol{v}(t, \boldsymbol{\sigma})$ defined by (1.9), and we have a solution of (1.4) and (1.5). We apply inverse Fourier transformation $\mathfrak{F}_{\sigma}^{-1}$ to $\boldsymbol{v}(t, \boldsymbol{\sigma})$ and have a solution of our Hukuhara's problem (1.1) and (1.2);

$$
\boldsymbol{u}(t, \boldsymbol{x})=\widetilde{F}_{\boldsymbol{\sigma}}^{-1} \boldsymbol{v}(t, \boldsymbol{\sigma}) \equiv \frac{1}{(2 \pi)^{m}} \int \exp (i \boldsymbol{\sigma} \boldsymbol{x}) \boldsymbol{v}(t, \boldsymbol{\sigma}) d \boldsymbol{\sigma} .
$$

Indeed, we have assumed in $\mathbb{A})$ ii) that the absolute values of $\hat{b}_{j}(t, \boldsymbol{\sigma})$ 's are bounded by some function of $\sigma$ independently of $t$. As is seen easily from the very way of construction of $v(t, \sigma)$ the same property is bequethed to $\partial_{t} v_{j}(t, \sigma)$ 's so that we can change the order of derivation and integration in differentiating the expression (1.24) with respect to $t$.

To sum up;

Theorem 1.1. Under the assumption A), (1.1) has a solution us $(t, x)$ defined for $0 \leqq t \leqq T_{0}$, which satisfies (1.2) for $0 \leqq t_{j} \leqq B_{0}$, $j=1,2, \cdots, N$ with a properly chosen constant $B_{0}>0$.

1.5. We shall state some remarks on stability and uniqueness of the solution constructed above.

i) Under the assumption $\mathbb{A}), \boldsymbol{v}(t, \boldsymbol{\sigma})$ is the unique solution of (1.4) and (1.5), which means the uniqueness of $u(t, x)$ in the class of soluticn to be obtained by the above procedure. That is to say, the solution $\mathbb{u}(t, x)$ obtained above is the unique solution of our problem (1.1) and (1.2) in the class of functions satisfying

$$
\begin{aligned}
& u_{j}(t, x) \in L_{x}^{1}, \\
& \left|\partial_{t} u_{j}(t, \boldsymbol{x})\right|<u_{0}(\boldsymbol{x}) \quad \text { with some } u_{0}(\boldsymbol{x}) \in L_{x}^{1}, \\
& \mathfrak{\mho}_{x} u_{j}(t, \boldsymbol{x}) \equiv v_{j}(t, \boldsymbol{\sigma}) \in L_{\boldsymbol{\sigma}}^{1},
\end{aligned}
$$




$$
\left|\partial_{t} v_{j}(t, \boldsymbol{\sigma})\right|<v_{0}(\boldsymbol{\sigma}) \quad \text { with some } \quad v_{0}(\boldsymbol{\sigma}) \in L_{\boldsymbol{\sigma}}^{1} .
$$

ii) Let $t_{j}^{(n)}, j=1,2, \cdots, N$ be converge to $t_{j}$ as $n \rightarrow \infty$. Corresponding to each crew $t_{j}^{(n)}, j=1,2, \cdots \mathrm{V}$ we have $\left.V\right|_{H^{(n)}}, \mathfrak{e}^{(n)}, \mathfrak{v}^{(n)}, \boldsymbol{u}^{(n)}$ etc. It can be easily seen that we have successively $\left.\left.V(t, \sigma)\right|_{H^{(n)}} \rightarrow V(t, \boldsymbol{\sigma})\right|_{H}, \mathbb{c}^{(n)}(\boldsymbol{\sigma})$ $\rightarrow \mathfrak{c}(\boldsymbol{\sigma}), \boldsymbol{v}^{(n)}(t, \boldsymbol{\sigma}) \rightarrow \boldsymbol{v}(t, \boldsymbol{\sigma}) \boldsymbol{u}^{(n)}(t, x) \rightarrow \boldsymbol{u}(t, \boldsymbol{x})$ uniformly as $n \rightarrow \infty$. This means the stability of the solution $\boldsymbol{u}(t, x)$ given by (1.24) with respect to the variation of $t_{j}$.

iii) Let $\varphi_{j}^{(n)}(x), j=1,2, \cdots, N$ be such functions that the Fourier transforms of them converge to $\hat{\varphi}_{j}(\sigma)$ in $L_{\sigma}^{1}$;

$$
\hat{\varphi}_{j}^{(n)}(\boldsymbol{\sigma}) \rightarrow \hat{\varphi}_{j}(\boldsymbol{\sigma}) \quad \text { in } \quad L_{\sigma}^{1} \quad \text { as } n \rightarrow \infty \text {. }
$$

Then we have successively

$$
\boldsymbol{v}^{(n)}(t, \boldsymbol{\sigma}) \rightarrow \boldsymbol{v}(t, \boldsymbol{\sigma}) \quad \text { in } \quad L_{\boldsymbol{\sigma}}^{1}, \quad \mathbb{a}^{(n)}(t, x) \rightarrow \mathfrak{e}(t, \boldsymbol{x}) \text { in } L_{\boldsymbol{x}}^{1},
$$

which means the stability of $\boldsymbol{u}(t, \boldsymbol{x})$ reith respect to the variation of $\boldsymbol{\varphi}(\boldsymbol{x})$ expressed in terms of its Fourier transform $\hat{\varphi}(\boldsymbol{\sigma})$.

\section{§2. A special case.}

2.1. The assumption A) ii) iii) can be weakened to some extent for differential equations of special type. Consider equations of the following type

$$
\left\{\begin{array}{l}
\partial_{t} u_{1}(t, \boldsymbol{x})=u_{2}(t, \boldsymbol{x})+b_{1}(t, \boldsymbol{x}), \\
\partial_{t} u_{2}(t, \boldsymbol{x})=\sum_{|\boldsymbol{k}| \leqq p} a_{\boldsymbol{k}}(t) \mathbf{D}^{\boldsymbol{k}} u_{1}(t, \boldsymbol{x})+b_{2}(t, \boldsymbol{x}) .
\end{array}\right.
$$

We shall solve Hukuhara's problem for this system, and find a solution of it, defined for $0 \leqq t \leqq T_{0}$ and satisfying the initial condition with Hukuhara's data

$$
u_{j}\left(t_{j}, x\right)=\varphi_{j}(\boldsymbol{x}), \quad j=1,2 .
$$

We assume;

B) i) $a_{k}(t)$ 's are defined for $0 \leqq t \leqq T_{0}$, are continuous, bounded, and satisfy

$$
\alpha_{0}(\boldsymbol{\sigma})^{2} \geqq \hat{a}(t, \boldsymbol{\sigma}) \equiv \sum_{|\boldsymbol{k}| \geqq p} a_{\boldsymbol{k}}(t) \boldsymbol{\sigma}^{k} \geqq 0 \quad \text { for } \quad|\boldsymbol{\sigma}| \geqq X_{0},
$$


with some constant $X_{0}$ and some continuous function $\alpha_{0}(\sigma)(\geqq 0)$. Without any restriction of generality, we can assume that $a_{0}(\boldsymbol{\sigma})=$ $O\left(|\boldsymbol{\sigma}|^{p / 2}\right)$ as $|\boldsymbol{\sigma}| \rightarrow \infty$.

ii) $b_{j}(t, \boldsymbol{x})$ 's are defined for $0 \leqq t \leqq T_{0}$, belong to $L_{x}^{1} . \hat{b}_{j}(t, \sigma)$ 's, the Fourier transforms with respect to $\boldsymbol{x}$ of $b_{j}(t, \sigma)$ 's are continuous with respect to the variable $t$, and satisfy $\left|\hat{b}_{j}(t, \sigma)\right| \leqq \beta_{0}(\sigma)$ with a function $\beta_{0}(\boldsymbol{\sigma})$ possessing the properties $\beta_{0}(\boldsymbol{\sigma})\left(|\boldsymbol{\sigma}|^{3 p / 2}+1\right) \in L_{\boldsymbol{\sigma}}^{1}$ and

$$
\begin{aligned}
\beta_{0}(\boldsymbol{\sigma})=O\left(\exp \left(-\left(T_{0}+\varepsilon\right) a_{0}(\boldsymbol{\sigma})\right)\right) & \text { with } \quad \varepsilon>0 \\
& \text { for }|\boldsymbol{\sigma}| \rightarrow \infty .
\end{aligned}
$$

iii) $\varphi_{j}(\boldsymbol{x})$ 's belong to $L_{\boldsymbol{x}}^{1} . \hat{\varphi}_{j}(\boldsymbol{\sigma})$ 's satisfy $\left|\hat{\varphi}_{j}(\boldsymbol{\sigma})\right| \leqq \varphi_{0}(\boldsymbol{\sigma})$ with a function $\varphi_{0}(\boldsymbol{\sigma})$ possessing the properties $\varphi_{0}(\boldsymbol{\sigma})\left(|\boldsymbol{\sigma}|^{p}+1\right) \in L_{\sigma}^{1}$ and

$$
\begin{array}{lll}
\varphi_{0}(\boldsymbol{\sigma})=O\left(\exp \left(-\left(T_{0}+\varepsilon^{\prime}\right) \alpha_{0}(\boldsymbol{\sigma})\right)\right) & \text { with } & \varepsilon^{\prime}>0 \\
& \text { for } \quad|\boldsymbol{\sigma}| \rightarrow \infty .
\end{array}
$$

2.2. Fourier transformation applied to (2.1) and (2.2) gives

$$
\begin{aligned}
& \left\{\begin{array}{l}
\partial_{t} v_{1}(t, \boldsymbol{\sigma})=v_{2}(t, \boldsymbol{\sigma})+\hat{b}_{1}(t, \boldsymbol{\sigma}), \\
\partial_{t} v_{2}(t, \boldsymbol{\sigma})=\hat{a}(t, \boldsymbol{\sigma}) v_{1}(t, \boldsymbol{\sigma})+\hat{b}_{2}(t, \boldsymbol{\sigma}),
\end{array}\right. \\
& v_{j}\left(t_{j}, \boldsymbol{\sigma}\right)=\varphi_{j}(\boldsymbol{\sigma}), \quad j=1,2 .
\end{aligned}
$$

As we have seen in $\S 1$, there is a solution $v(t, \sigma)$ of (2.6) defined for $0 \leqq t \leqq T_{\mathrm{c}}$ and $|\boldsymbol{\sigma}| \leqq X_{0}$, satisfying (2.7) for $0 \leqq t_{j} \leqq B_{0}$ with a properly chosen $B_{0}$. We shall investigate (2.6) and (2.7) when $|\sigma|>X_{0}$.

Let $\boldsymbol{v}^{(j)}(t, \boldsymbol{\sigma}), j=1,2$ be a fundamental system of solution of the reduced equations

$$
\left\{\begin{array}{l}
\partial_{t} v_{1}(t, \sigma)=v_{2}(t, \sigma), \\
\partial_{2} v_{2}(t, \sigma)=\hat{a}(t, \sigma) v_{1}(t, \sigma),
\end{array}\right.
$$

satisfying the initial conditions

$$
\boldsymbol{v}^{(1)}(0, \boldsymbol{\sigma})=\left(\begin{array}{l}
1 \\
1
\end{array}\right), \quad \boldsymbol{v}^{(2)}(0, \boldsymbol{\sigma})=\left(\begin{array}{l}
0 \\
1
\end{array}\right)
$$

It is easily seen that the components $v_{j}^{(1)}(t, \sigma), j=1,2$ of the vector $\boldsymbol{v}^{(1)}(t, \boldsymbol{\sigma})$ satisfy $v_{j}^{(1)}(t, \boldsymbol{\sigma}) \geqq 1$ and are monotone increasing as 
$t$ increases. Exact form of the solution $\boldsymbol{v}^{(2)}(t, \boldsymbol{\sigma})$ expressed by $\boldsymbol{v}_{j}^{(1)}(t, \boldsymbol{\sigma})$ can be obtained by the method of depression of the order;

$$
\left\{\begin{array}{l}
v_{1}^{(2)}(t, \boldsymbol{\sigma})=v_{1}^{(1)}(t, \boldsymbol{\sigma}) \int_{0}^{t} \frac{d s}{\left(v_{1}^{(1)}(s, \boldsymbol{\sigma})\right)^{2}} \\
v_{2}^{(2)}(t, \boldsymbol{\sigma})=v_{2}^{(1)}(t, \boldsymbol{\sigma}) \int_{0}^{t} \frac{d s}{\left(\tau_{1}^{(1)}(s, \boldsymbol{\sigma})\right)^{2}}+\frac{1}{v_{1}^{(1)}(t, \boldsymbol{\sigma})} .
\end{array}\right.
$$

The Wronskian of these solutions $V(t, \boldsymbol{\sigma})=\left(\boldsymbol{v}^{(1)}(t, \boldsymbol{\sigma}), \boldsymbol{v}^{(2)}(t, \boldsymbol{\sigma})\right)$ can be calculated easily;

$$
\operatorname{det} V(t, \sigma) \equiv \text { const. }=\operatorname{det} V(0, \sigma)=1 .
$$

As for the Hukuhara's determinant, we have

$$
\begin{aligned}
& \operatorname{det}\left(\left.V(t, \sigma)\right|_{H}\right)=\left|\begin{array}{ll}
\tau_{1}^{(1)}\left(t_{1}, \sigma\right) & v_{1}^{(2)}\left(t_{1}, \sigma\right) \\
\tau_{2}^{(1)}\left(t_{2}, \sigma\right) & v_{2}^{(2)}\left(t_{2}, \sigma\right)
\end{array}\right| \\
& =\left|\begin{array}{ll}
v_{1}^{(1)}\left(t_{1}, \boldsymbol{\sigma}\right) & \tau_{1}^{(1)}\left(t_{1}, \boldsymbol{\sigma}\right) \int_{0}^{t_{1}} \frac{d s}{\left(v_{1}^{(1)}(s, \sigma)\right)^{2}} \\
v_{2}^{(1)}\left(t_{2}, \boldsymbol{\sigma}\right) & v_{2}^{(1)}\left(t_{2}, \boldsymbol{\sigma}\right) \int_{0}^{t_{2}} \frac{d s}{\left(v_{1}^{(1)}(s, \sigma)\right)^{2}}+\frac{1}{v_{1}^{(1)}\left(t_{2}, \boldsymbol{\sigma}\right)}
\end{array}\right| \\
& =\tau_{1}^{(1)}\left(t_{1}, \sigma\right)\left(r_{2}^{(1)}\left(t_{2}, \sigma\right) \int_{t_{1}}^{t_{2}} \frac{d s}{\left(v_{1}^{(1)}(s, \sigma)\right)^{2}}+\frac{1}{v_{1}^{(1)}\left(t_{2}, \sigma\right)}\right) \\
& \geqq v_{1}^{(1)}\left(t_{1}, \boldsymbol{\sigma}\right)\left(\int_{t_{1}}^{t_{2}} \frac{\tau_{2}^{(1)}(s, \boldsymbol{\sigma})}{\left(v_{1}^{(1)}(s, \boldsymbol{\sigma})\right)^{2}} d s+\frac{1}{v_{1}^{(1)}\left(t_{2}, \boldsymbol{\sigma}\right)}\right) \\
& =v_{1}^{(1)}\left(t_{1}, \boldsymbol{\sigma}\right)\left(\left[\frac{-1}{v_{1}^{(1)}(s, \sigma)}\right]_{t_{1}}^{t_{2}}+\frac{1}{v_{1}^{(1)}\left(t_{2}, \sigma\right)}\right)=1 \text {. }
\end{aligned}
$$

2.3. To obtain an estimation of $\boldsymbol{v}^{(1)}(t, \boldsymbol{\sigma})$, we consider equations

$$
\left\{\begin{array}{l}
\partial_{t} w_{1}^{(\delta)}(t, \sigma)=w_{2}^{(\delta)}(t, \sigma), \\
\partial_{t} w_{2}^{(\delta)}(t, \sigma)=\left(\alpha_{0}(\boldsymbol{\sigma})+\delta\right)^{2} w_{1}^{(\delta)}(t, \boldsymbol{\sigma}), \text { with a parameter } \delta>0,
\end{array}\right.
$$

together with initial conditions

$$
\left\{\begin{array}{l}
w_{1}^{(\delta)}(0, \boldsymbol{\sigma})=1+1 /\left(\alpha_{0}(\boldsymbol{\sigma})+\boldsymbol{\delta}\right), \\
w_{2}^{(\delta)}(0, \boldsymbol{\sigma})=1+\alpha_{0}(\boldsymbol{\sigma})+\delta .
\end{array}\right.
$$

(2.11) and (2.12) have the unique solution

$$
\left\{\begin{array}{l}
w_{1}^{(\delta)}(t, \boldsymbol{\sigma})=\left(1+1 /\left(\alpha_{0}(\boldsymbol{\sigma})+\delta\right)\right) \exp \left(\left(\alpha_{0}(\boldsymbol{\sigma})+\delta\right) t\right), \\
w_{2}^{(\delta)}(t, \boldsymbol{\sigma})=\left(1+\alpha_{0}(\boldsymbol{\sigma})+\delta\right) \exp \left(\left(\alpha_{0}(\boldsymbol{\sigma})+\boldsymbol{\delta}\right) t\right),
\end{array}\right.
$$


for which we have

$$
\begin{aligned}
& v_{j}^{(1)}(0, \boldsymbol{\sigma})<w_{j}^{(\delta)}(0, \sigma), j=1,2, \\
& \partial_{t} v_{j}^{(1)}(t, \sigma)<\partial_{t} w_{\sigma}^{(\delta)}(\dot{i}, \boldsymbol{\sigma}) \text { as long as } v_{l}^{(1)}(t, \widetilde{\sigma})<w_{l}^{(\delta)}(t, \sigma)
\end{aligned}
$$

$l \neq j ; j=1,2$.

From these relations we can easily deduce

$$
v_{j}^{(1)}(t, \boldsymbol{\sigma})<w_{j}^{(\delta)}(t, \boldsymbol{\sigma}) \text { for } t \geqq 0, j=1,2,
$$

and as the limit of $\delta \rightarrow 0$ we have

$$
\left\{\begin{array}{l}
1 \leqq v_{1}^{(1)}(t, \sigma) \leqq\left(1+1 / \alpha_{0}(\sigma)\right) \exp \left(\alpha_{0}(\boldsymbol{\sigma}) t\right), \\
1 \leqq v_{2}^{(1)}(t, \sigma) \leqq\left(1+\alpha_{0}(\boldsymbol{\sigma})\right) \exp \left(\alpha_{0}(\boldsymbol{\sigma}) t\right),
\end{array}\right.
$$

which, together with (2.9), gives

$$
\left\{\begin{aligned}
0 \leqq v_{1}^{(2)}(t, \sigma) & =v_{1}^{(1)}(t, \sigma) \int_{0}^{t} \frac{d s}{\left(v_{1}^{(1)}(s, \sigma)\right)^{2}} \leqq t v_{1}^{(1)}(\dot{t}, \boldsymbol{\sigma}) \\
& \leqq t\left(1+1 / \alpha_{0}(\boldsymbol{\sigma})\right) \exp \left(\alpha_{0}(\boldsymbol{\sigma}) t\right), \\
1 \leqq v_{2}^{(2)}(t, \sigma) & =v_{2}^{(1)}(t, \sigma) \int_{0}^{t} \frac{d s}{\left(v_{1}^{(1)}(s, \sigma)\right)^{2}}+\frac{1}{v_{1}^{(1)}(t, \sigma)} \\
& <t v_{1}^{(1)}(t, \sigma)+1<t\left(1+\alpha_{0}(\boldsymbol{\sigma}) \exp \left(\alpha_{0}(\boldsymbol{\sigma}) t\right)\right)+1
\end{aligned}\right.
$$

2. 4. We take up a particular solution $\boldsymbol{v}^{(0)}(t, \sigma)$ of the inhomogeneous equations (2.6) given by the formula

$$
\boldsymbol{v}^{(0)}(t, \boldsymbol{\sigma})=V(t, \boldsymbol{\sigma}) \int_{0}^{t}(V(s, \boldsymbol{\sigma}))^{-1 \hat{b}}(s, \boldsymbol{\sigma}) d s,
$$

in which we used the notations

$$
\begin{aligned}
& V(t, \boldsymbol{\sigma})=\left(\begin{array}{cc}
\tau_{1}^{(1)}(t, \boldsymbol{\sigma}) & v_{1}^{(2)}(t, \boldsymbol{\sigma}) \\
v_{2}^{(1)}(t, \boldsymbol{\sigma}) & v_{2}^{(2)}(t, \boldsymbol{\sigma})
\end{array}\right), \\
& (V(s, \boldsymbol{\sigma}))^{-1}=\left(\begin{array}{rr}
v_{2}^{(2)}(s, \boldsymbol{\sigma}) & -v_{1}^{(2)}(s, \boldsymbol{\sigma}) \\
-v_{2}^{(1)}(s, \boldsymbol{\sigma}) & v_{1}^{(1)}(s, \boldsymbol{\sigma})
\end{array}\right) .
\end{aligned}
$$

Since each column vector $w^{(j)}(t, s, \boldsymbol{\sigma}), j=1,2$ of the matrix $W(t, s, \boldsymbol{\sigma})$ $\equiv V(t, \sigma)(V(s, \sigma))^{-1}, 0 \leqq s \leqq t \leqq T_{0}$ satisfies the equations (2.8) together with the initial condition $\boldsymbol{w}^{(1)}(s, s, \boldsymbol{\sigma})=\left(\begin{array}{l}1 \\ 0\end{array}\right)$ or $\boldsymbol{w}^{(2)}(s, s, \boldsymbol{\sigma})=\left(\begin{array}{l}0 \\ 1\end{array}\right)$, it can be expressed as a linear combination of fundamental solutions. 
From (2.17) and (2.18) we know that the absolute values of the elements of $V(t, \boldsymbol{\sigma})(V(s, \boldsymbol{\sigma}))^{-1}, 0 \leqq s \leqq t \leqq T_{0}$ do not exceed $\gamma\left(\exp \left(\alpha_{0}(\boldsymbol{\sigma}) t\right)\right)$. The coefficient $r=\gamma\left(t, a_{0}(\sigma)\right)$ can be taken so as to be monotone increasing with respect to $t$ and satisfy

$$
\gamma\left(t, \alpha_{0}\right)=O\left(\alpha_{0}\right) \text {, as } \quad \tilde{c}_{0} \rightarrow \infty .
$$

From (2.19) and (2.20) we have

$$
\left|\gamma_{j}^{(0)}(t, \boldsymbol{\sigma})\right| \leqq t r \beta_{0}(\boldsymbol{\sigma}) \exp \left(\alpha_{0}(\boldsymbol{\sigma}) t\right), \text { for } 0 \leqq t \leqq T_{0} .
$$

Especially we have

$$
\left|v_{j}^{(0)}\left(t_{j}, \boldsymbol{\sigma}\right)\right| \leqq B_{0} \gamma^{\prime} \beta_{0}(\boldsymbol{\sigma}) \exp \left(\boldsymbol{a}_{0}(\boldsymbol{\sigma}) \beta_{0}\right), \text { for } 0 \leqq t_{j} \leqq B_{0}\left(\leqq T_{0}\right)
$$

with $\gamma^{\prime}=\gamma\left(\beta_{0}, \alpha_{0}(\boldsymbol{\sigma})\right)$.

2.5. We shall find a solution $v(t, \boldsymbol{\sigma})$ of (2.6) and (2.7) for $|\boldsymbol{\sigma}|>X_{\mathrm{c}}$ in the form of

$$
\boldsymbol{v}(t, \boldsymbol{\sigma})=\boldsymbol{v}^{(0)}(t, \boldsymbol{\sigma})+V(t, \boldsymbol{\sigma}) \boldsymbol{c}(\boldsymbol{\sigma}), \quad \boldsymbol{c}(\boldsymbol{\sigma})=\left(\begin{array}{c}
c_{1}(\boldsymbol{\sigma}) \\
c_{2}(\boldsymbol{\sigma})
\end{array}\right) .
$$

From (2.7) we have

$$
\left.\boldsymbol{v}(t, \boldsymbol{\sigma})\right|_{H}=\left.\boldsymbol{v}^{(v)}(t, \boldsymbol{\sigma})\right|_{H}+\left.V(t, \boldsymbol{\sigma})\right|_{H} \boldsymbol{C}(\boldsymbol{\sigma})=\hat{\boldsymbol{\varphi}}(\boldsymbol{\sigma}) .
$$

As we have seen in 2.2, det $\left(\left.V(t, \sigma)\right|_{H}\right) \neq 0$ and we can solve (2.24) with respect to $\boldsymbol{e}(\boldsymbol{\sigma})$;

$$
\boldsymbol{c}(\boldsymbol{\sigma})=\left(\left.V(t, \boldsymbol{\sigma})\right|_{H}\right)^{-1}\left(\hat{\boldsymbol{\varphi}}(\boldsymbol{\sigma})-\left.\boldsymbol{v}^{(0)}(t, \boldsymbol{\sigma})\right|_{H}\right) .
$$

The elements of the matrix $\left(\left.V(t, \sigma)\right|_{H}\right)^{-1}$ can be estimated by help of (2.10), (2.17) and (2.18), and we have from (2.25)

$$
\left|c_{j}(\boldsymbol{\sigma})\right|<\gamma^{\prime \prime} \varphi_{0}(\boldsymbol{\sigma}) \exp \left(\alpha_{0}(\boldsymbol{\sigma}) B_{0}\right)+\gamma^{\prime \prime \prime} \beta_{0}(\boldsymbol{\sigma}) \exp \left(2 \alpha_{0}(\boldsymbol{\sigma}) B_{0}\right),
$$

in which $\gamma^{\prime \prime}$ and $\gamma^{\prime \prime \prime}$ depend on both $T_{0}$ and $\alpha_{0}(\boldsymbol{\sigma})$, and $\gamma^{\prime \prime}=O\left(\alpha_{0}\right)$, $r^{\prime \prime \prime}=O\left(\alpha_{0}^{2}\right)$ as $\alpha_{0} \rightarrow \infty$. Since we have estimated all the quantities appearing in the second member of (2.23), we have at last

$$
\begin{gathered}
\left|\gamma_{j}(t, \boldsymbol{\sigma})\right| \leqq \gamma^{\mathrm{IV}} \beta_{0}(\boldsymbol{\sigma})\left\{\exp \left(\alpha_{0}(\boldsymbol{\sigma}) T_{0}\right)+\exp \left(\alpha_{0}(\boldsymbol{\sigma})\left(2 B_{0}+T_{0}\right)\right)\right\} \\
+\gamma^{\mathrm{v}} \varphi_{0}(\boldsymbol{\sigma}) \exp \left(\alpha_{0}(\boldsymbol{\sigma})\left(B_{0}+T_{0}\right)\right),
\end{gathered}
$$

in which $\gamma^{\mathrm{IV}}$ and $\gamma^{\mathrm{V}}$ depend on bath $T_{0}$ and $\alpha_{0}(\boldsymbol{\sigma}), \gamma^{\mathrm{IV}}=O\left(\alpha_{0}^{3}\right), \gamma^{V}=O\left(a_{0}^{2}\right)$ 
as $\alpha_{0} \rightarrow \infty$.

For any value of the constant $B_{0}\left(\leqq T_{0}\right)$ taken so small as to satisfy

$$
\left\{\begin{array}{l}
2 B_{0}<T_{0}+\varepsilon \\
B_{0}<\varepsilon^{\prime}
\end{array}\right.
$$

we can find a positive constant $\varepsilon_{0}$ for which we have

$$
\left|v_{j}(t, \sigma)\right| \leqq \gamma_{0} \exp \left(-\varepsilon_{0} \alpha_{0}(\sigma)\right) \text {, for }|\sigma|>X_{0},
$$

with some constant $\gamma_{0}$ depending on $T_{0}$ and $\alpha_{0}(\boldsymbol{\sigma})$ and $\gamma_{0}=O\left(\alpha_{0}^{3}\right)$ as $\alpha_{0} \rightarrow \infty$. We have seen already in 2.2 that a solution $v(t, \boldsymbol{\theta})$ of $(2.6)$ and (2.7) exists for $|0| \leqq X_{0}$. We have thus accomplished the construction of $v(t, \mathbb{E})$ for any value of the parameter.

From (2.27) (2.29) and the assumptions $\mathbb{B}$ ) ii) iii), we know that $v_{j}(t, \sigma)$ 's belong to $L_{\boldsymbol{\sigma}}^{1}$. We have a solution $x_{\ell}(t, x)$ of $(2.1)$ and (2.2) by inverse Fourier transformation;

$$
\mathbb{u}(t, x)=\mathfrak{F}_{\sigma}^{-1} \boldsymbol{v}(t, \boldsymbol{\sigma}) \equiv \frac{1}{(2 \pi)^{n}} \int \exp (i \boldsymbol{\sigma} x) \boldsymbol{v}(t, \boldsymbol{\sigma}) d \boldsymbol{\sigma} .
$$

To sum up;

Thearem 2.1。 Under the assumption 留), (2.1) has a solution ue $(t, x)$ ciefined for $0 \leqq t \leqq T_{0}$, which satisfies (2.2) for $0 \leqq t_{j} \leqq B_{0}$, $j=1,2$, with a properily chosen consiant $\bar{b}_{0}$.

2.6. The remarks on iniqueness and stability of solution stated in $\mathbb{1}$. 5 , remain still valid with an evident aiteration that the assumption A) should be replaced by the assumption $\mathbb{B}$ ) in this case. Also the condition " $\hat{\varphi}_{j}^{(n)}(\sigma) \rightarrow \hat{\varphi}_{j}(\sigma)$ in $L_{\sigma}^{1}$ as $n \rightarrow \infty$ " stated there, becomes " $\left(|\sigma|^{p}+1\right) \hat{\varphi}_{j}^{(n)}(\sigma) \rightarrow\left(|\sigma|^{p}+1\right) \hat{\varphi}_{j}(\sigma)$ in $L_{\sigma}^{1}$ as $n \rightarrow \infty$ " in this case.

\section{§3. Convolution equations.}

3. 1. Hukuhara's problem for convolution equations can be treated in the same manner as in the preceding sections. Consider the following convolution equations

$$
\partial_{t} \boldsymbol{u}(t, \boldsymbol{x})=A(t, \boldsymbol{x}) * \boldsymbol{u}(t, \boldsymbol{x})+\boldsymbol{b}(t, \boldsymbol{x}),
$$


in which $A(t, x)$ means an $N \times N$ matrix and the symbol $*$ means convolution with respect to the variables $x=\left(x_{1}, x_{2}, \cdots, x_{m}\right)$. We shall solve Hukuhara's problem for (3.1), and find a solution of it defined for $0 \leqq t \leqq T_{0}$ and satisfying the initial condition with Hukuhara's data

$$
u_{j}\left(t_{j}, \boldsymbol{x}\right)=\varphi_{j}(\boldsymbol{x}), j=1,2, \cdots, N,
$$

or in vector form

$$
\left.\boldsymbol{u}(t, \boldsymbol{x})\right|_{H}=\varphi(x) .
$$

Fourier transformation with respect to $x$, applied to (3.1) and (3. $\left.2^{\prime}\right)$ gives

$$
\begin{aligned}
& \partial_{t} \boldsymbol{v}(t, \boldsymbol{\sigma})=\hat{A}(t, \boldsymbol{\sigma}) \boldsymbol{v}(t, \boldsymbol{\sigma})+\hat{\boldsymbol{\theta}}(t, \boldsymbol{\sigma}), \\
& \left.\boldsymbol{v}(t, \boldsymbol{\sigma})\right|_{H}=\hat{\boldsymbol{\varphi}}(\boldsymbol{\sigma}) .
\end{aligned}
$$

We assume

C) i) The elements $a_{j l}(t, x)$ 's of the matrix $A(t, x)$ cire defined for $0 \leqq t \leqq T_{0}$, belong to $L_{\boldsymbol{x}}^{1}$. Their Fourier iransforms $\hat{a}_{j l}(i, \infty)$ 's are continuous with respeci to $t$ and belong to $L_{\sigma}^{1}$, and satisfy $\left|\hat{\alpha}_{j}(t, \sigma)\right| \leqq x_{0}$ for $0 \leqq t \leqq T_{0}$ with a constant $\alpha_{0}>0$.

ii) The components $b,(t, x)$ 's of the recior. $B(t, x)$ aire Giefined for

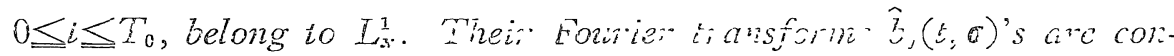

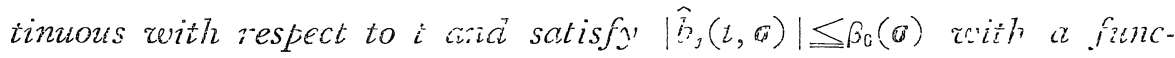
tion $\Theta_{0}(\otimes)$ belonging to $L_{\sigma}^{1}$.

iii) The components $\varphi_{j}(x)$ 's of the rector $\theta(x)$ belong to $L_{\alpha}^{1}$. Their Fourier trarsforms $\hat{\varphi}_{j}(\sigma)$ 's belong to $L_{\sigma}^{1}$.

3. 2. Consider the reduced equations of (3.3);

$$
\partial_{t} \boldsymbol{v}(t, \boldsymbol{\sigma})=\hat{A}(t, \boldsymbol{\sigma}) \boldsymbol{v}(t, \boldsymbol{\sigma}) .
$$

From the assumption $\mathbb{C}$ ) i) we know that we can treat (3.5) in the same manner as (1.6) in $\S 1$, but without any restriction on the value of $\boldsymbol{\sigma}$. There is a fundamental system of solutions $V(t, \boldsymbol{\sigma})=\left(\boldsymbol{v}^{(1)}(t, \boldsymbol{\sigma})\right.$, $\left.\boldsymbol{v}^{(2)}(t, \boldsymbol{\sigma}), \cdots, \boldsymbol{v}^{(N)}(t, \boldsymbol{\sigma})\right)$ which satisfies

$$
\operatorname{det}\left(\left.V(t, \boldsymbol{\sigma})\right|_{H}\right) \geqq 1 / 2 \text { for } 0 \leqq t_{j} \leqq B_{0}\left(\leqq T_{0}\right),
$$


with a sufficiently small constant $B_{0}$.

A particular solution $\boldsymbol{v}^{(0)}(t, \sigma)$ of the equations (3.3) is given in the form

$$
\boldsymbol{v}^{(0)}(t, \boldsymbol{\sigma})=V(t, \boldsymbol{\sigma}) \int_{0}^{t}(\boldsymbol{v}(s, \boldsymbol{\sigma}))^{-1 \hat{\mathbf{B}}}(s, \boldsymbol{\sigma}) d s,
$$

which is bounded and belongs to $L_{\sigma}^{1}$.

We shall find a solution $v(t, \boldsymbol{\theta})$ of (3.3) and (3.4) expressed in the form

$$
\boldsymbol{v}(t, \boldsymbol{\sigma})=\boldsymbol{v}^{(0)}(t, \boldsymbol{\sigma})+V(t, \boldsymbol{\sigma}) \mathfrak{e}(\boldsymbol{\sigma}), \boldsymbol{c}(\boldsymbol{\sigma})=\left(\begin{array}{c}
c_{1}(\boldsymbol{\sigma}) \\
c_{2}(\boldsymbol{\sigma}) \\
\cdots \\
c_{N}(\boldsymbol{\sigma})
\end{array}\right)
$$

From (3.4) we have

$$
\mathscr{C}(\boldsymbol{\sigma})=\left(\left.V(t, \boldsymbol{\sigma})\right|_{H}\right)^{-1}\left(\hat{\boldsymbol{\varphi}}(\boldsymbol{\sigma})-\left.\boldsymbol{v}^{(0)}(t, \boldsymbol{\sigma})\right|_{H}\right),
$$

which, substituted in (3.8), gives the desired solution $v(t, \boldsymbol{\sigma})$ belonging to $L_{\boldsymbol{\sigma}}^{1}$. Inverse Fourier transformation applied to $\boldsymbol{v}(t, \boldsymbol{\sigma})$ gives a solution $\boldsymbol{u}(t, \boldsymbol{x})$ of our Hukuhara's problem (3.1) and (3.2);

$$
\mathbb{u}(t, \boldsymbol{x})=\mathfrak{F}_{\boldsymbol{\sigma}}^{-1} \boldsymbol{v}(t, \boldsymbol{\sigma}) \equiv \frac{1}{(2 \pi)^{m}} \int \exp (i \boldsymbol{\sigma} \boldsymbol{x}) \boldsymbol{v}(t, \boldsymbol{\sigma}) \partial \boldsymbol{\sigma} .
$$

To sum up;

Theorem 3.1. Under the assumption $\mathbf{C}$ ), (3.1) has a solution $\boldsymbol{u}(t, \boldsymbol{x})$ defined for $0 \leqq t \leqq T_{0}$, which satisfies (3.2) for $0 \leqq t_{j} \leqq B_{0}$ with a properly chosen constant $B_{0}>0$.

The remarks on uniqueness and stability of solution stated in the preceding sections, remain still valid in this case.

3. 3. The assumption C) i) can be weakened to some extent for convolution equations of special type. Analogous circumstances have occured for differential equations (1.1) and (2.1) in the preceding sections.

Consider convolution equations of the following type;

$$
\left\{\begin{array}{l}
\partial_{t} u_{1}(t, \boldsymbol{x})=u_{2}(t, \boldsymbol{x})+b_{1}(t, \boldsymbol{x}) \\
\partial_{t} u_{2}(t, \boldsymbol{x})=a(t, \boldsymbol{x}) * u_{1}(t, \boldsymbol{x})+b_{2}(t, \boldsymbol{x}) .
\end{array}\right.
$$


We shall find a solution $\boldsymbol{u}(t, \boldsymbol{x})$ of (3.11) defined for $0 \leqq t \leqq T_{0}$, satisfying the condition

$$
u_{j}\left(t_{j}, \boldsymbol{x}\right)=\varphi_{j}(\boldsymbol{x}), j=1,2 .
$$

Fourier transformation with respect to $\boldsymbol{x}$ applied to (3.11) and (3. 12) gives

$$
\begin{aligned}
& \left\{\begin{array}{l}
\partial_{t} v_{1}(t, \boldsymbol{\sigma})=v_{2}(t, \boldsymbol{\sigma})+\hat{b}_{1}(t, \boldsymbol{\sigma}), \\
\partial_{t} v_{2}(t, \boldsymbol{\sigma})=\hat{a}(t, \boldsymbol{\sigma}) v_{1}(t, \boldsymbol{\sigma})+\hat{b}_{2}(t, \boldsymbol{\sigma}),
\end{array}\right. \\
& v_{j}\left(t_{j}, \boldsymbol{\sigma}\right)=\hat{\varphi}_{j}(\boldsymbol{\sigma}), \quad j=1,2,
\end{aligned}
$$

or in vector form

$$
\left.\boldsymbol{v}(t, \boldsymbol{\sigma})\right|_{H}=\hat{\boldsymbol{\varphi}}(\boldsymbol{\sigma}) .
$$

We assume:

D) i) The function $a(t, \boldsymbol{x})$ is defined for $0 \leqq t \leqq T_{0}$, belongs to $L_{\boldsymbol{x}}^{1}$. Its Fourier transform $\hat{a}(t, \sigma)$ is continuous rith respect to $t$, belongs to $L_{\boldsymbol{\sigma}}^{1}$, and satisfies

$$
\alpha_{0}(\boldsymbol{\sigma})^{2} \geqq \hat{a}(t, \boldsymbol{\sigma}) \geqq 0, \text { for }|\boldsymbol{\sigma}|>X_{0},
$$

with a constant $X_{0} \geqq 0$ and a continuous function $\alpha_{0}(\boldsymbol{\sigma})$, and

$$
\alpha_{1} \geqq|\hat{a}(t, \sigma)|, \text { for } \quad|\boldsymbol{\sigma}| \leqq X_{0},
$$

with a constant $\alpha_{1}$.

ii) The components $b_{j}(t, \boldsymbol{x})$ 's of the rector $\boldsymbol{b}(t, \boldsymbol{x})$ are defined for $0 \leqq t \leqq T_{0}$, belong to $L_{\boldsymbol{x}}^{1}$. Their Fourier transforms $\hat{b}_{j}(t, \boldsymbol{\sigma})$ 's are continuous with respect to $t$ and satisfy $\left|\hat{b}_{i}(t, \sigma)\right| \leqq \beta_{0}(\boldsymbol{\sigma})$ with a function $\beta_{0}(\boldsymbol{\sigma})$ possessing the properties $\left(\alpha_{0}(\boldsymbol{\sigma})^{3}+1\right) \beta_{0}(\boldsymbol{\sigma}) \in L_{\boldsymbol{\sigma}}^{1}$ and $\beta_{0}(\boldsymbol{\sigma})=$ $O\left(\exp \left(-\left(T_{0}+\varepsilon\right) \alpha_{0}(\boldsymbol{\sigma})\right)\right), \varepsilon>0$, for $|\boldsymbol{\sigma}| \rightarrow \infty$.

iii) The components $\varphi_{j}(\boldsymbol{x})^{\prime}$ 's of the vector $\boldsymbol{\varphi}(\boldsymbol{x})$ belong to $L_{\boldsymbol{x}}^{1}$. The Fourier transforms $\hat{\varphi}_{j}(\boldsymbol{\sigma})$ 's satisfy $\left|\hat{\varphi}_{j}(\boldsymbol{\sigma})\right| \leqq \varphi_{0}(\boldsymbol{\sigma})$ with a function $\varphi_{0}(\boldsymbol{\sigma})$ possessing the properties $\left(\alpha_{0}(\boldsymbol{\sigma})^{2}+1\right) \varphi_{0}(\boldsymbol{\sigma}) \in L_{\boldsymbol{\sigma}}^{1}$ and $\varphi_{\mathrm{c}}(\boldsymbol{\sigma})=$ $O\left(\exp \left(-\left(T_{0}+\varepsilon^{\prime}\right) \alpha_{0}(\boldsymbol{\sigma})\right)\right)$ with $\varepsilon^{\prime}>0$ for $|\boldsymbol{\sigma}| \rightarrow \infty$.

3. 4. Under the assumption $\mathbf{D}),(3.13)$ and (3.14) can be treated in the same manner as in $\S 2$. There is a solution $v(t, \boldsymbol{\sigma})$ of (3.13) de- 
fined for $0 \leqq t \leqq T_{0}$ and satisfying (3.14) for $0 \leqq t_{j} \leqq B_{0}$ with a properly chosen constant $B_{0}>0$. Inverse Fourier transformation $\widetilde{F}_{\sigma}^{-1}$ applied to $\boldsymbol{v}(\boldsymbol{t}, \boldsymbol{\sigma})$ gives a solution $\boldsymbol{u}(t, \boldsymbol{x})$ of (3.11) satisfying (3.12).

The remarks on uniqueness and stability of solution remain still valid in this case with some obvious changes similar to the one given in $\mathbf{2 . 6}$ of $\$ 2$.

\section{§4. Semi-separate hyperbolice equations.}

First we quote from Nagumo [8] well known results concerning Cauchy problem for symmetric hyperbolic equations. Consider symmetric hyperbolic system

$$
\begin{aligned}
\Phi u(t, x) & \equiv \partial_{t} u \mathbb{u}(t, x)+\sum_{l=1}^{m} A_{l}(t, x) \partial_{l} \mathbb{u}(t, x)+B(t, x) u(t, x) \\
& =\boldsymbol{f}(t, x),
\end{aligned}
$$

together with a usual initial condition

$$
u(0, x)=\varphi(x) .
$$

The matrices $A_{l}(t, x)$ 's are symmetric; $A_{l}(t, x)=\left(a_{l j k}(t, x)\right)_{j, k=1,2, \ldots, N}$, $a_{l j k}(t, \boldsymbol{x})=a_{l k j}(t, \boldsymbol{x}), \quad B^{\prime}(t, \boldsymbol{x})$ is not necessarily so;

$$
B(t, x)=\left(b_{j k}(t, x)\right)_{j, k=1,2, \ldots, N .} .
$$

(4.1) is taken in generalized sense, that is to say, a solution $\boldsymbol{x}(t, x)$ is such a vector function that it belongs to $L_{(t, x)}^{2}$ on a domain $D(D$ in $(t, x)$ space and satisfies

$$
\begin{aligned}
& \left(\boldsymbol{u}, \Phi^{*} \boldsymbol{v}\right)=(\boldsymbol{f}, \boldsymbol{w})\left(\equiv \int_{j=1}^{N} f_{j}(t, \boldsymbol{x}) w_{j}(t, \boldsymbol{x}) d t d \boldsymbol{x}\right) \\
& \text { for all } \boldsymbol{w}(t, \boldsymbol{x})=\left(\begin{array}{c}
w_{1}(t, \boldsymbol{x}) \\
w_{2}(t, \boldsymbol{x}) \\
\vdots \\
w_{N}(t, \boldsymbol{x})
\end{array}\right), \quad w_{j}(t, \boldsymbol{x}) \in C_{0}^{\infty}(\mathfrak{D}),
\end{aligned}
$$

in which $\varpi^{*}$ means the adjoint operator of $\emptyset$.

We shall make use of the following theorems (Nagumo loc cit. pp. 70-71).

Theorem I. Let the elements of the matrices $A_{j}(t, \boldsymbol{x}), \partial_{l} A_{j}(t, \boldsymbol{x})$, 
$\left(t=0,1, \cdots, m ; \partial_{0}=\partial / \partial t\right)$ and $B(t, \boldsymbol{x})$ be bounded continuous functions defined on $(t, x)$-space $E^{n+1}$, Let the components $f_{j}(t, x)$ 's of the vector $\boldsymbol{f}(t, x)$ belong to $L_{(t, x)}^{2}$ on $E^{+1}$, and let the components $\varphi_{j}(\boldsymbol{x})$ of the vector $\varphi(x)$ belong to $L_{x}^{2}$ on $x$-space $E^{n}$. (Here after we prefer to say shortly " $\boldsymbol{f}(t, \boldsymbol{x})$ belongs to $L_{(t, \boldsymbol{x})}^{2}$ ", " $\boldsymbol{\varphi ( \boldsymbol { x } )}$ belongs to $L_{\boldsymbol{x}}^{2}$ " etc.)

For any fixed value $T_{0}>0$, there is a generalized solution $\boldsymbol{u}(t, \boldsymbol{x})$ of (4.1) defined for $0 \leqq t \leqq T_{0}$, satisfying (4.2) as an element of $L_{x}^{2}$. For fixed the components of $u(t, x)$ and $\Phi u(t, x)$ belong to $L_{x}^{2}$. Under this additional condition, solution of (4.1) and (4.2) is determined uniquely for $0 \leqq t \leqq T_{0}, x \in E^{n}$.

Theorem II. (Energy integral inquality) The solution $\boldsymbol{u}(t, \boldsymbol{x})$ of (4.1) and (4.2) given in Theorem I satisfies

$$
\|\boldsymbol{u}(t)\|^{2}+\left\|\mathbb{e d}_{\mathbb{e}}^{2}\right\|_{\left(0, T_{0}\right)} \leqq c_{T_{0}}\left(\left\|\boldsymbol{u}\left(t_{0}\right)\right\|^{2}+\|\boldsymbol{f}\|_{\left(0, T_{0}\right)}^{2}\right),
$$

in rohich the constant $c_{T_{0}}$ depends on $T_{0}$, and $c_{T_{0}} \rightarrow 1$ as $T_{0} \rightarrow 0$.

In the above formulation we used the notations

$$
\|\mathbb{e}(i)\|^{2}=\int_{j=1}^{N}\left(u_{j}(t, \approx x)^{2}\right) d x, \quad\|\mathbb{u}\|_{\left(0, T_{0}\right)}^{2}=\int_{0}^{T_{0}}\|\mathbb{\varepsilon}(t)\|^{2} d t .
$$

4.1. Consider the following equations

$$
\begin{aligned}
\partial_{i} \boldsymbol{u}_{j}(t, x) & +\sum_{i=1}^{m} A_{j l}(t, x) \partial_{l} \mathbb{u}_{j}(t, x)+B_{j}(t, x) \boldsymbol{u}_{j}(t, x) \\
& +\sum_{k \neq j} c_{j k}(t, x) \boldsymbol{u}_{k}(t, x)=\boldsymbol{f}_{j}(t, x), \quad j=1,2, \cdots, N,
\end{aligned}
$$

or in concise form

$$
\tilde{\Phi} \tilde{u}+\tilde{C} \tilde{u}=\tilde{f}
$$

in which we used the notations

$$
\tilde{\boldsymbol{u}}=\left(\begin{array}{c}
\boldsymbol{u}_{1} \\
\boldsymbol{u}_{2} \\
\vdots \\
\boldsymbol{u}_{N}
\end{array}\right), \quad \boldsymbol{u}_{j}=\left(\begin{array}{c}
u_{j 1} \\
u_{j 2} \\
\vdots \\
u_{j N_{j}}
\end{array}\right), \quad \boldsymbol{f}=\left(\begin{array}{c}
\boldsymbol{f}_{1} \\
\boldsymbol{f}_{2} \\
\vdots \\
\boldsymbol{f}_{N}
\end{array}\right), \quad \boldsymbol{f}_{j}=\left(\begin{array}{c}
f_{j 1} \\
f_{j 2} \\
\vdots \\
f_{j N_{j}}
\end{array}\right),
$$

$A_{j l}(t, x)^{\prime} s$ are symmetric $N_{j} \times N_{j}$ matrices, $B_{j}(t, x)$ 's are $N_{j} \times N_{j}$ matrices, $C_{j k}(t, \boldsymbol{x})^{\prime} s$ are $N_{j} \times N_{k}$ matrices.

We shall set up Hukuhara's problem for the equations (4.4) and 
find out a solution in generalized sense, defined for $0 \leqq t \leqq T_{0}$, satisfying the condition

$$
\boldsymbol{u}_{j}\left(t_{j}, \boldsymbol{x}\right)=\boldsymbol{\varphi}_{j}(\boldsymbol{x}) \quad \text { in } \quad L_{\boldsymbol{x}}^{2}, \quad j=1,2, \cdots, N,
$$

that is, every component of the vector $\boldsymbol{u}_{j}$ belong to $L_{\boldsymbol{x}}^{2}$ and equal to the corresponding component of the vector $\boldsymbol{\varphi}_{j}$ as an element of $L_{\boldsymbol{x}}^{2}$. (4.5) is written in the consice form

$$
\left.\widetilde{\boldsymbol{u}}\right|_{\tilde{H}}=\tilde{\boldsymbol{\varphi}} \text { in } L_{x}^{2},
$$

in which we used the notations

$$
\tilde{\varphi}_{(x)}=\left(\begin{array}{c}
\varphi_{1}(x) \\
\varphi_{2}(x) \\
\vdots \\
\varphi_{N}(x)
\end{array}\right), \quad \varphi_{j}(x)=\left(\begin{array}{c}
\varphi_{j 1}(x) \\
\varphi_{j 2}(x) \\
\vdots \\
\varphi_{j N}(x)
\end{array}\right)
$$

We assume;

E) i) The elements of the matrices $A_{j k}(t, x)$ 's, $\partial_{l} A_{j k}(t, x)$ 's $l=$ $0,1,2, \cdots, m, B_{j}(t, \boldsymbol{x})$ 's and $C_{j k}(t, \boldsymbol{x})$ 's are bounded continuous functions of $(t, x)$. Moreover, the elements $c_{j k h l}(t, x)$ 's of the matrices $C_{j k}(t, x)$ 's satisfy $\left|c_{j k h l}(t, \boldsymbol{x})\right| \leqq c_{0}$ with such a constant $c_{0}$ that it satisfies

$$
(N-1) c_{0}^{2}\left(\max _{j} N_{j}^{2}\right)<1 .
$$

ii) $\boldsymbol{f}_{j}(t, \boldsymbol{x})$ 's belong to $L_{(\mathrm{t}, x)}^{2}$ in $E^{n+1} \cdot \boldsymbol{\varphi}_{j}(\boldsymbol{x})^{\text {'s }}$ belong to $L_{\boldsymbol{x}}^{2}$ in $E^{n}$.

4. 2. We put $\tilde{\boldsymbol{u}}^{(0)}(t, \boldsymbol{x}) \equiv \mathbf{0} \equiv\left(\begin{array}{c}0 \\ 0 \\ \vdots \\ 0\end{array}\right)$, and define $\left\{\tilde{\boldsymbol{u}}^{(n)}(t, \boldsymbol{x})\right\}_{n=1}^{\infty}$ inductively as solutions of the following Hukuhara's problem

$$
\begin{aligned}
& \tilde{\Phi} \tilde{\boldsymbol{u}}^{(n+1)}=\tilde{\boldsymbol{f}}-\tilde{C} \tilde{\boldsymbol{u}}^{(n)}, \\
& \left.\tilde{\boldsymbol{u}}^{(n+1)}\right|_{\tilde{H}}=\tilde{\boldsymbol{\varphi}}, \quad n=1,2, \cdots .
\end{aligned}
$$

(4.7) and (4.8) can be separated into $N$ Cauchy problems with respect to $\mathbb{u}_{j}^{(n+1)}, j=1,2, \cdots, N$. From thecrem I we know that $\tilde{u}^{(n)}(t, x)$ exists and is unique for $0 \leqq t \leqq T_{0}$ with any $T_{0}>0, n=1,2, \cdots$.

From theorem II we have

$$
\left\|\boldsymbol{u} \boldsymbol{u}_{j}^{(1)}(t)\right\|^{2}+\left\|\boldsymbol{E \boldsymbol { E } _ { j } ^ { ( ? ) }}\right\|_{\left(0, T_{0}\right.}^{2} \leqq C_{j T_{0}}\left(\left\|\boldsymbol{\varphi}_{j}\right\|^{2}+\left\|\boldsymbol{f}_{j}\right\|_{\left(0 . T_{0}\right)}^{2}\right),
$$




$$
j=1,2, \cdots, N \text {. }
$$

Using the notations $\left\|\tilde{\boldsymbol{u}}_{(\mathrm{t})}^{(1)}\right\|^{2}=\sum_{j=1}^{N}\left\|\boldsymbol{u}_{j(\mathrm{t})}^{(1)}\right\|^{2}, \quad\|\tilde{\boldsymbol{\varphi}}\|^{2}=\sum_{j=1}^{N}\left\|\boldsymbol{\varphi}_{j}\right\|^{2}$ etc., we have

$$
\left\|\tilde{\boldsymbol{u}}_{(\mathrm{t})}^{(1)}\right\|^{2}+\left\|\tilde{\boldsymbol{u}}^{(1)}\right\|_{\left(0, T_{0}\right)} \leqq c_{T_{0}}^{\prime}\left(\|\tilde{\boldsymbol{\varphi}}\|^{2}+\|\tilde{\boldsymbol{f}}\|_{\left(0, T_{0}\right)}^{2}\right)
$$

with $c_{T_{0}}^{\prime}=\max _{j} c_{j T_{0}}$.

For general value of $n$ we have

$$
\begin{aligned}
& \tilde{\Phi}\left(\tilde{\boldsymbol{u}}^{(n+1)}-\tilde{\boldsymbol{u}}^{(n)}\right)=-\tilde{C}\left(\tilde{\boldsymbol{u}}^{(n)}-\tilde{\boldsymbol{u}}^{(n-1)}\right), \\
&\left.\left(\tilde{\boldsymbol{u}}^{(n+1)}-\tilde{\boldsymbol{u}}^{(n)}\right)\right|_{\tilde{H}}=\tilde{\mathbf{0}} .
\end{aligned}
$$

Application of Theorem II to $\boldsymbol{u}_{j}^{(n+1)}-\boldsymbol{u}_{j}^{(n)}$ gives

$$
\begin{aligned}
& \left\|u_{j}^{(n+1)}(t)-\boldsymbol{u}_{j}^{(n)}(t)\right\|^{2}+\left\|\boldsymbol{u}_{j}^{(n+1)}-u_{j}^{(n)}\right\|_{\left(0, T_{0}\right)}^{2} \\
& \leqq c_{j T_{0}}\left\|\sum_{k \neq j} C_{k}\left(\boldsymbol{u}_{k}^{(n)}-\boldsymbol{u}_{k}^{(n-1)}\right)\right\|_{\left(0, T_{0}\right)}^{2} \\
& \leqq c_{j T_{0}} \sum_{k \neq j} c_{0}^{2} N_{k}^{2}\left\|\boldsymbol{u}_{k}^{(n)}-\boldsymbol{u}_{k}^{(n-1)}\right\|_{\left(0, T_{0}\right)}^{2},
\end{aligned}
$$

and

$$
\begin{aligned}
& \left\|\tilde{\boldsymbol{u}}_{(t)}^{(n+1)}-\tilde{\boldsymbol{u}}_{(t)}^{(n)}\right\|^{2}+\left\|\tilde{\boldsymbol{u}}^{(n+1)}-\tilde{\boldsymbol{u}}^{(n)}\right\|_{\left(0, T_{0}\right)}^{2} \\
& \leqq \sum_{j=1}^{N} c_{j T_{0}} \sum_{k \neq j} c_{0}^{2} N_{k}^{2}\left\|\boldsymbol{e}_{k}^{(n)}-\boldsymbol{u}_{k}^{(n-1)}\right\|_{\left(0, T_{0}\right)}^{2} \\
& \leqq c_{T_{0}}^{\prime} c_{0}^{2}(N-1)\left(\max _{j} N_{j}^{2}\right)\left\|\tilde{\boldsymbol{u}}^{(n)}-\tilde{\boldsymbol{u}}^{(n-1)}\right\|_{\left(0, T_{0}\right)}^{2} .
\end{aligned}
$$

Since we can let $c_{j T_{0}} \rightarrow 1$ as $T_{0} \rightarrow 0$, we can take

$$
c_{T_{0}}^{\prime \prime} \equiv c_{T_{0}}^{\prime} c_{0}^{2}(N-1)\left(\max _{j} N_{j}^{2}\right)<1,
$$

for sufficiently small $T_{0}$, and we have inductively

$$
\begin{aligned}
& \left\|\tilde{\boldsymbol{u}}^{(n+1)}-\tilde{\boldsymbol{u}}^{(n)}\right\|_{\left(0, T_{0}\right)}^{2} \leqq c_{T_{0}}^{\prime \prime n}\left\|\tilde{\boldsymbol{u}}^{(1)}\right\|_{\left(0, T_{0}\right)}^{2} \leqq c_{T_{0}}^{\prime} c_{T_{0}}^{\prime \prime n}\left(\|\tilde{\boldsymbol{\varphi}}\|^{2}+\|\tilde{\boldsymbol{f}}\|_{\left(0, T_{0}\right)}^{2}\right), \\
& \left\|\tilde{\boldsymbol{u}}_{(t)}^{(n+1)}-\boldsymbol{u}_{(t)}^{(n)}\right\|^{2} \leqq c_{T_{0}}^{\prime \prime n}\left\|\tilde{\boldsymbol{u}}^{(1)}\right\|_{\left(0, T_{0}\right)}^{2} \leqq c_{T_{0}}^{\prime} c_{T_{0}}^{\prime \prime n}\left(\|\tilde{\boldsymbol{\varphi}}\|^{2}+\|\tilde{\boldsymbol{f}}\|_{\left(0, T_{0}\right)}^{2}\right) .
\end{aligned}
$$

From (4.16) and (4.17) we have convergence in $L_{(t, \boldsymbol{x})}^{2}$ of $\tilde{\boldsymbol{u}}_{(t, \boldsymbol{x})}^{(n)}$ and convergence in $L_{\boldsymbol{x}}^{2}$ for fixed $t$ of $\tilde{\boldsymbol{u}}_{(t, \boldsymbol{x})}^{(n)}$, and from (4.7) also convergence in $L_{x}^{2}$ for fixed $t$ of $\widetilde{\Phi} \widetilde{u}^{(n)}(t, x)$. Hence we have a solution $\widetilde{z}(t, x)$ of (4.7) and (4.8) defined as the limit of $\tilde{a}^{(n)}(t, x)$ as $n \rightarrow \infty$ for $0 \leqq t \leqq T_{0}$ with sufficiently small $T_{0}>0$.

To sum up, 
Theorem 4.1. Under the assumption $\mathbb{E}$ ) there is a generalized solution $\widetilde{\boldsymbol{u}}(t, \boldsymbol{x})$ of $(4.4)$ defined for $0 \leqq t \leqq T_{0}$ with a properly chosen $T_{0}>0$ and satisfying (4.5) for $0 \leqq t_{j} \leqq T_{0}$.

4. 3. The solution $\widetilde{u}(t, x)$ obtained above is unique and stable with respect to the variation of $t_{j}$ 's and $\varphi_{j}$ 's.

Indeed, let $\tilde{u}(t, x)$ and $\tilde{\boldsymbol{v}}(t, x)$ be two solutions of (4.4) and (4.5) given for $0 \leqq t \leqq T_{0}$ with a constant $T_{0}$ satisfying (4.15) under the assumption $\mathbb{E}$ ). By the same argument as the one used to derive (4.14), we have

(4. 18) $\quad\|\tilde{\mathbb{U}}(t)-\tilde{\mathfrak{v}}(t)\|^{2}+\|\tilde{\mathbb{U}}-\tilde{\mathfrak{v}}\|_{\left(0, T_{0}\right)}^{2}<c_{T_{0}}^{\prime \prime}\|\tilde{\mathbb{E}}-\tilde{\mathfrak{v}}\|_{\left(0, T_{0}\right)}^{2}$,

which gives $\widetilde{u}(t, x)=\tilde{\mathfrak{v}}(t, x)$ in $L_{(t, x)}^{2}$ or uniqueness of solution for $0 \leqq t \leqq T_{0}$, since we have $c_{T_{0}}^{\prime \prime}<1$ by (4.15).

Stability of solution with respect to the variation of $\tilde{\phi}(x)$ is obtained similarly. Let $\widetilde{\varepsilon}(t, x)$ and $\tilde{\mathcal{v}}(t, x)$ be the solutions of (4.4) and (4.5) corresponding to the data $\tilde{\varphi}(x)$ and $\tilde{\psi}(x)$ respectively, defined for $0 \leqq t \leqq T_{0}$ with a constant $T_{0}$ satisfying (4.15) under the assumption $\mathbb{E})$. After the manner of (4.18) we have

$$
\|\tilde{\mathbb{E}}(t)-\tilde{\mathbb{W}}(t)\|^{2}+\left(1-c_{T_{0}}^{\prime \prime}\right)\|\tilde{\mathbb{Z}}-\tilde{\mathbb{W}}\|_{\left(0, T_{0}\right)}<c_{T_{0}}^{\prime \prime}\|\tilde{\varphi}-\tilde{\psi}\|^{2},
$$

which gives the convergence of $\tilde{v}(t, x)$ to $\tilde{x}(t, x)$ in both $L_{x}^{2}$ and $L_{(t, x)}^{2}$ as $\tilde{\psi}(x)$ converges to $\tilde{\varphi}(x)$ in $L_{x}^{2}$.

To investigate stability of solution with respect to the variation of $t_{j}$ 's, let $\tilde{\mathbb{u}}(t, x)$ and $\tilde{\mathfrak{v}}(t, x)$ be the solutions of (4.4) defined for $0 \leqq t \leqq T_{0}$ and satisfying

$$
\boldsymbol{u}_{j}\left(t_{j}, \boldsymbol{x}\right)=\varphi_{j}(x)
$$

or

$$
v_{j}\left(s_{j}, \boldsymbol{x}\right)=\varphi_{j}(\boldsymbol{x}),
$$

with $\left|t_{j}-s_{j}\right| \leqq \delta, j=1,2, \cdots, N$, respectively, under the assumption $\mathbb{E}$ ). We assume in addition, $\left.\mathbb{E}^{\prime}\right)$ The components $\varphi_{j k}(x)$ of the vector $\tilde{\varphi}(x)$ have derivatives in generalized sense belonging to $L_{x}^{2}$; $\partial_{l} \varphi_{j k}(\boldsymbol{x}) \in L_{\boldsymbol{x}}^{2}$.

From $\left(\left(4.20^{\prime}\right)\right.$ and $\left.\mathbb{E}^{\prime}\right)$ we have 


$$
\begin{aligned}
& \Phi_{j}\left(\boldsymbol{v}_{j}-\boldsymbol{\varphi}_{j}\right)+\sum_{k \neq j} C_{j k} \boldsymbol{v}_{k}=\boldsymbol{f}_{j}(t, \boldsymbol{x})-\Phi_{j} \boldsymbol{\varphi}_{J}, \\
& \boldsymbol{v}_{j}\left(s_{j}, \boldsymbol{x}\right)-\boldsymbol{\varphi}_{j}(\boldsymbol{x})=0 .
\end{aligned}
$$

Since $\|\tilde{\boldsymbol{v}}\|_{\left(0, T_{0}\right)}$ is finite, we can apply Theorem II to $\boldsymbol{v}_{j}(t, \boldsymbol{x})-\boldsymbol{\varphi}_{j}(\boldsymbol{x})$ for $t_{j}-\delta \leqq t \leqq t_{j}+\delta$ and obtain

$$
\left\|\boldsymbol{v}_{j}(t)-\varphi_{j}\right\|^{2} \leqq c_{\delta}\left\|\boldsymbol{f}_{j}-\Phi_{j} \varphi_{j}-\sum_{k \neq j} \boldsymbol{v}_{k}\right\|_{\left(t_{j}-\delta, t_{j}+\delta\right)}^{2}
$$

with a constant $c_{\delta}$, which remains bounded as $\delta \rightarrow 0$. From (4.23) we have $v_{j}\left(t_{j}, x\right) \rightarrow \varphi_{j}(x)=\mathbb{u}_{j}\left(t_{j}, x\right)$ in $L_{\boldsymbol{x}}^{2}$ as $s_{j} \rightarrow t_{j}, j=1,2, \cdots, N$, from which it follows $\tilde{\boldsymbol{v}}(t, \boldsymbol{x}) \rightarrow \tilde{\boldsymbol{E}}(t, \boldsymbol{x})$ in $L_{(t, \boldsymbol{x})}^{2}$ as we have seen already.

It is well known that starting from the energy integral inequality (4.3), one can deal with genuine solution of Cauchy problem and Cauchy problem for semilinear system. But it is inmediately seen that an energy integral inequality for the system (4.4) can be given in the same form as (4.3), using the simple method by which we have derived (4.14) from (4.13). Therefore it seems to be unnecessary to state here the same results for Hukuhara's problem as the well known ones for Cauchy problem concerning semilinear system or gunune solution.

\section{REFERENCES}

[1] K. Akô, Semi-decomposable systems and vector subfunctions, Funk. Ekv.. 8 (1966), 91-97.

[2] M. Hukuhara, Sur les singurarités des fonctions définies par des équations différentielles II. Japan. J. Math., 8 (1931), 143-158.

[3] M. Hukuhara, La propriété de Kneser globale et le probléme aux limites, Publ. Research. Inst. Math. Sci., Kyoto Univ., Ser. A, 1 (1966), 129-148.

[4] T. Kusano, On the boundary problem for quasilinear systems for parabolic differential equations in non-cylindrical domains, Funk. Ekv., 7 (1965), 163-178.

[5] T. Kusano, Periodic solutions of the first boundary problem for quasilinear parabolic equations of the second order, (to appear).

[6] S. Mizohata, Partial differential equations (in Japanese), Tokyo, 1965.

[7] M. Nagumo, Degree of mapping and existence theorems (in Japanese), Tokyo, 1948.

[8] M. Nagumo, Modern theory of partial differential equations (in Japanese), Tokyo, 1957.

[9] A. Plis, Generalization of the Cauchy problem to a system of partial differential equations, Bull. Acad. Polon. Sci., 4 (1956), 741-744.

[10] Z. Szmydt, Sur un nouveau type de problèmes pour un système d'équations dif- 
férentielles hyperboliques du second ordre a deux variables indépendantes, ibid., 4 (1956), 67-72.

[11] S. Yosida, Hukuhara's problem for partial differential equations, Funk, Ekv., 8 (1965), 5-37.

[12] S. Yosida, Boundary value problem with Hukuhara's data for parabolic systems. ibid., 9 (1966), 61-69. 